\title{
Application of infrared microspectroscopy and multivariate analysis for monitoring the effect of adjunct cultures during Swiss cheese ripening
}

\author{
G. Chen, ${ }^{1}$ N. A. Kocaoglu-Vurma, ${ }^{1}$ W. J. Harper, and L. E. Rodriguez-Saona ${ }^{2}$ \\ Department of Food Science and Technology, The Ohio State University, Columbus 43210
}

\begin{abstract}
Improved cheese flavor has been attributed to the addition of adjunct cultures, which provide certain key enzymes for proteolysis and affect the dynamics of starter and nonstarter cultures. Infrared microspectroscopy provides unique fingerprint-like spectra for cheese samples and allows for rapid monitoring of cheese composition during ripening. The objective was to use infrared microspectroscopy and multivariate analysis to evaluate the effect of adjunct cultures on Swiss cheeses during ripening. Swiss cheeses, manufactured using a commercial starter culture combination and 1 of 3 adjunct Lactobacillus spp., were evaluated at d $1,6,30,60$, and 90 of ripening. Cheese samples (approximately $20 \mathrm{~g}$ ) were powdered with liquid nitrogen and homogenized using water and organic solvents, and the water-soluble components were separated. A $3-\mu \mathrm{L}$ aliquot of the extract was applied onto a reflective microscope slide, vacuum-dried, and analyzed by infrared microspectroscopy. The infrared spectra (900 to $1,800 \mathrm{~cm}^{-1}$ ) produced specific absorption profiles that allowed for discrimination among different cheese samples. Cheeses manufactured with adjunct cultures showed more uniform and consistent spectral profiles, leading to the formation of tight clusters by patternrecognition analysis (soft independent modeling of class analogy) as compared with cheeses with no adjuncts, which exhibited more spectral variability among replicated samples. In addition, the soft independent modeling of class analogy discriminating power indicated that cheeses were differentiated predominantly based on the band at $1,122 \mathrm{~cm}^{-1}$, which was associated with $\mathrm{S}-\mathrm{O}$ vibrations. The greatest changes in the chemical profile of each cheese occurred between d 6 and 30 of warm-room ripening. The band at $1,412 \mathrm{~cm}^{-1}$, which was associated with acidic AA, had the greatest contribution to differentiation, indicating substantial changes in levels of proteolysis during warm-room ripening in
\end{abstract}

\footnotetext{
Received December 1, 2008.

Accepted March 12, 2009.

${ }^{1}$ Both authors contributed equally to this work.

${ }^{2}$ Corresponding author: Rodriguez-saona.1@osu.edu
}

addition to propionic acid, acetic acid, and eye formation. A high-throughput infrared microspectroscopy technique was developed that can further the understanding of biochemical changes occurring during the ripening process and provide insight into the role of adjunct nonstarter lactic acid bacteria on the complex process of flavor development in cheeses.

Key words: Swiss cheese, infrared, chemometrics, adjunct culture

\section{INTRODUCTION}

Swiss cheese is a hard cheese characterized by its sweet, nutty flavor and the presence of eyes caused by the production of carbon dioxide from lactate fermentation by propionibacteria (Langsrud and Reinbold, 1973; Noël et al., 1999). Cheese flavor that develops during ripening plays an important role in determining the cheese quality (Singh et al., 2003). Cheese ripening or maturation involves a series of complex microbiological, physical, and chemical changes, including proteolysis, glycolysis, and lipolysis, which lead to changes in cheese composition, and thereby to the development of unique texture and flavor characteristics (Fox et al., 2000). Proteolysis and the consequent derivatives of AA influence flavor development during cheese ripening (Fox et al., 1994; Law, 2001; Gorostiza et al., 2004).

Crow et al. (2001) reported that nonstarter lactic acid bacteria (NSLAB) are the main uncontrolled factor in current industrial cheese manufacture. On the other hand, the intentional addition of NSLAB as adjunct cultures has found application in the cheese industry because some NSLAB possess key enzymes that accelerate proteolysis for cheese flavor development (Kieronczyk et al., 2003), and the addition of adjunct cultures can indirectly control the secondary microflora in cheese (Crow et al., 2001). Lynch et al. (1997) reported that when compared with control cheeses, cheese manufactured with adjunct lactobacilli showed better flavor intensity and higher levels of free AA, probably because of increased proteolysis caused by growth of the nonstarter cultures.

The increase in total production and the demand for cheeses of high and consistent quality (Woodcock et 
al., 2008) require the presence of an easy and rapid examination method to help cheese manufacturers better understand the effect of adjunct cultures during cheese ripening and for the production of high- and consistent-quality products. Conventional cheese composition analysis is time-consuming and expensive and requires the use of hazardous organic solvents, which limit the application of these procedures in the dairy industry to monitor real-time composition during ripening (Gorostiza et al., 2004). Infrared spectroscopy is a sensitive tool that provides unique fingerprint-like spectra for cheese samples (Chen and Irudayaraj, 1998) and can allow for rapid monitoring of cheese composition during the ripening time (Martin-del-Campo et al., 2007). Fourier-transform infrared microspectroscopy has been used for the characterization and identification of microorganisms (Wenning et al., 2002; Ngo-Thi et al., 2003). Infrared microspectroscopy combines 2 analytical technologies for biological analysis by coupling an infinity-corrected microscope with a high-performance infrared spectrometer, providing capabilities for high-throughput screening of microorganisms and the ability to resolve spectral profiles within desired regions of the target (Männig et al., 2008). Infrared microspectroscopy has permitted the acquisition of spectra from samples as small as $100 \mathrm{pg}\left(10^{-10} \mathrm{~g}\right)$, thus promoting its application in the medical and biological fields (Diem et al., 2004). Infrared microspectroscopy can allow for monitoring of subtle changes in cheese composition, thus providing valuable insights into the complex biochemical changes occurring during cheese ripening. The objective of this study was to use infrared microspectroscopy and chemometrics to investigate the effect of adjunct Lactobacillus spp. on Swiss cheese during ripening.

\section{MATERIALS AND METHODS}

\section{Cheese Samples}

Swiss cheese samples (duplicates of 4 treatments), manufactured by using a modified rindless block procedure (Kocaoglu-Vurma et al., 2008) and 1 of 3 adjunct Lactobacillus spp. (Lactobacillus casei A26, L. casei B21, or Lactobacillus rhamnosus H2) or no adjunct, were studied. After brining, cheeses were stored in a cold room $\left(4\right.$ to $\left.7^{\circ} \mathrm{C}\right)$ for the first $6 \mathrm{~d}$ to allow for salt equilibration throughout the cheese (initial cooling). After cold-room storage, cheeses were transferred to a warm room $\left(21\right.$ to $\left.22^{\circ} \mathrm{C}\right)$ for $24 \mathrm{~d}$ for eye development, and then back to the cold room $\left(4\right.$ to $\left.7^{\circ} \mathrm{C}\right)$ for further ripening. Cheeses were sampled at d 1 (before brining), d 6 (end of initial cooling), d 30 (end of warm-room rip- ening), and d 60 and 90 (cold-room ripening). Cheese samples (approximately $200 \mathrm{~g}$ ) were vacuum-packaged in 3-mil nylon-polyethylene standard barrier vacuum pouches, stored at $-80^{\circ} \mathrm{C}$, and randomized for unbiased analysis before sample preparation and analysis.

\section{Sample Preparation}

Swiss cheese extracts were prepared as described by Subramanian et al. (2009). Frozen cheese samples (approximately $20 \mathrm{~g}$ ) were powdered with liquid nitrogen, and approximately $0.1 \mathrm{~g}$ of powdered cheese sample was mixed with $0.5 \mathrm{~mL}$ of deionized water in a $1.5-\mathrm{mL}$ centrifugation tube. The mixture was sonicated (Sonic Dismembrator Model 100, Fisher, Pittsburgh, PA) with an output of $8 \mathrm{~W}$ for $10 \mathrm{~s}$, and chloroform $(0.5$ $\mathrm{mL}$, Fisher) was added. The mixture was homogenized by vortexing, and then centrifuged at $16,100 \times g$ for $3.5 \mathrm{~min}$ at room temperature (Microcentrifuge Model 5415R, Eppendorf, Westbury, NY). An aliquot (200 $\mu \mathrm{L}$ ) of the supernatant was collected, mixed with an equal volume of ethanol (ethyl alcohol, Acros Organics, Fisher), and centrifuged again at 16,100 $\times g$ for 3.5 min to precipitate residual proteins and polypeptides. A $3-\mu \mathrm{L}$ aliquot of the extract was applied onto a reflective microscope slide (SpectCONC-IR low-e MirrIR microscope slide, Tienta Science, Indianapolis, IN) and vacuum-dried in a desiccator for approximately $5 \mathrm{~min}$ at room temperature.

\section{Infrared Microspectroscopy}

Measurements were performed on a Varian 600 UMA Fourier-transform infrared microscope (Varian, Randolph, MA). The infrared microscope was interfaced with an FTS Excalibur 3100GX Fourier-transform infrared spectrometer (Varian) equipped with a dynamically aligned Michelson interferometer, a motorized $\mathrm{x}-\mathrm{y}$ stage, and $4 \times$ and $16 \times$ objectives. The instrument had a Permaglow (ceramic) midinfrared source, an extended-range potassium bromide beam splitter, and a liquid nitrogen-cooled mercury cadmium telluride infrared detector. Spectra were acquired using Win-IR Pro Software (version 3.4.2, Varian Inc., Palo Alto, CA). The infrared spectra were obtained from 4,000 and $700 \mathrm{~cm}^{-1}$ with a resolution of $8 \mathrm{~cm}^{-1}$, and 128 scans were co-added per spectrum to enhance the signal-tonoise ratio. A smooth, gold-coated metal was used for background measurement because gold has no interfering absorption under the midinfrared radiation region. For each cheese sample, powders (20 g) were prepared in triplicate. Cheese powders $(0.1 \mathrm{~g})$ were extracted in duplicate and 3 spectra were collected from each 
extract, giving a total of 18 spectra per cheese sample. The cheeses were manufactured in duplicate, resulting in a total of 36 spectral measurements per treatment at each ripening time.

\section{Multivariate Analysis}

Multivariate training models (classification models) were generated using soft independent modeling of class analogy (SIMCA) by Pirouette software (version 3.11, InfoMetrix Inc., Woodville, WA). The Fouriertransform infrared spectral data were mean-centered and transformed to their second derivative using a 5-point Savitzky-Golay polynomial algorithm, and the vector length was normalized.

Soft independent modeling of class analogy is a pattern-recognition method based on principal components analysis that reduces the dimensionality in multivariate data so that any systematic variation present may be investigated (Lavine, 2000). Training sets are assigned to classes and a principal components model is generated for each class, with distinct confidence regions within them (De Maesschalck et al., 1999). The variance that is explained by the class model describes the signal, whereas the noise in the data is described by the residual variance. The number of principal components retained in the model is determined by using a crossvalidation procedure that accounts for most of the variation within each class, ensuring high signal to noise by not including the noise-laden principal components in the class model (Lavine, 2000). The scores plot allows the visualization of clustering among samples (sample patterns, groupings, or outliers). Sample residual and Mahalanobis distance (De Maesschalck et al., 2000) were used for outlier diagnostics. The discriminating power in SIMCA provides a diagnostic tool that indicates, on a relative scale, how important a wavenumber in the spectrum is to differentiating classes. The SIMCA analysis assesses itself by predicting each sample included in the training set, comparing that prediction to its assigned class; this assessment is referred to as misclassification. Zero misclassifications typify a model in which all samples were correctly predicted to the preassigned class. A 3-dimensional score plot projection based on the first 3 principal components was used to visualize the separation of classes. Interclass distances (ICD) were calculated using between-class residuals, and greater ICD indicated a greater difference between the clusters (Kvalheim and Karstang, 1992; Dunn and Wold, 1995). Variable importance, also known as discriminating power, was used to define the spectral regions that had a predominant effect on classification of the cheese sample.

\section{RESULTS AND DISCUSSION}

A representative raw spectrum and its second derivative from the water-soluble fraction of Swiss cheese manufactured with each adjunct strain are shown in Figure 1. Mathematical processing of the raw spectra using a second-derivative algorithm (5-point gap size) allowed the resolution of overlapping bands and the removal of baseline variation, and also provided high discriminating potential (Schmitt and Flemming, 1998; Kansiz et al., 2001). Rodriguez-Saona et al. (2006) previously reported that the nonuniform distribution of the eye on the Swiss cheese surface and the strong infrared absorption of major components such as protein and lipids limits the detection of minor components. Thus, extracting the water-soluble fraction from the cheese has been shown to improve the detection and quantification capabilities of infrared spectroscopy (Koca et al., 2007; Subramanian et al., 2009). The water-soluble components in cheese include AA, lactones, aldehydes, ketones, and sulfur-containing compounds (Engels et al., 1997). Major absorption bands (Figure 1) were observed at $856,926,1,045,1,122,1,315$, and 1,416 $\mathrm{cm}^{-1}$ that have been associated with $\mathrm{C}-\mathrm{H}$ out-of-plane bending on an aromatic ring (Coates, 2000), O-H outof-plane bending of carboxylic acids (Smith, 1998), C-O stretching of alcohols (Coates, 2000), stretching vibrations of S-O bands (Sun and Wang, 2006), C-O stretching of carboxylic acids (Smith, 1998; Safar et al., 1994), and $\mathrm{O}-\mathrm{C}-\mathrm{O}$ stretching vibrations of carboxylates (Byler and Farrell, 1989), respectively. The spectral region at 1,800 to $900 \mathrm{~cm}^{-1}$ was used for the classification and analysis of Swiss cheese samples by adjunct cultures.

\section{The Effect of Adjunct Cultures}

Swiss cheese manufactured with 3 different adjunct Lactobacillus spp. strains and manufactured with no adjunct cultures were studied at d 1 (before brining), d 6 (end of initial cooling), d 30 (end of warm-room ripening), and d 60 and 90 (cold-room ripening). Subtle spectral differences were observed among cheese samples manufactured with different adjunct cultures (Figure 2). Soft independent modeling of class analogy analysis of the second-derivative Fourier-transform infrared spectra allowed classification of the Swiss cheese samples according to their ripening stage. The SIMCA projection plot (Figure 3A), a 3-dimensional visual image based on the first 3 principal components, showed that by using the infrared spectral range from 1,800 to $900 \mathrm{~cm}^{-1}$, Swiss cheese samples were well separated into different clusters after $90 \mathrm{~d}$ of ripening according to the adjunct cultures used during manufacture. 


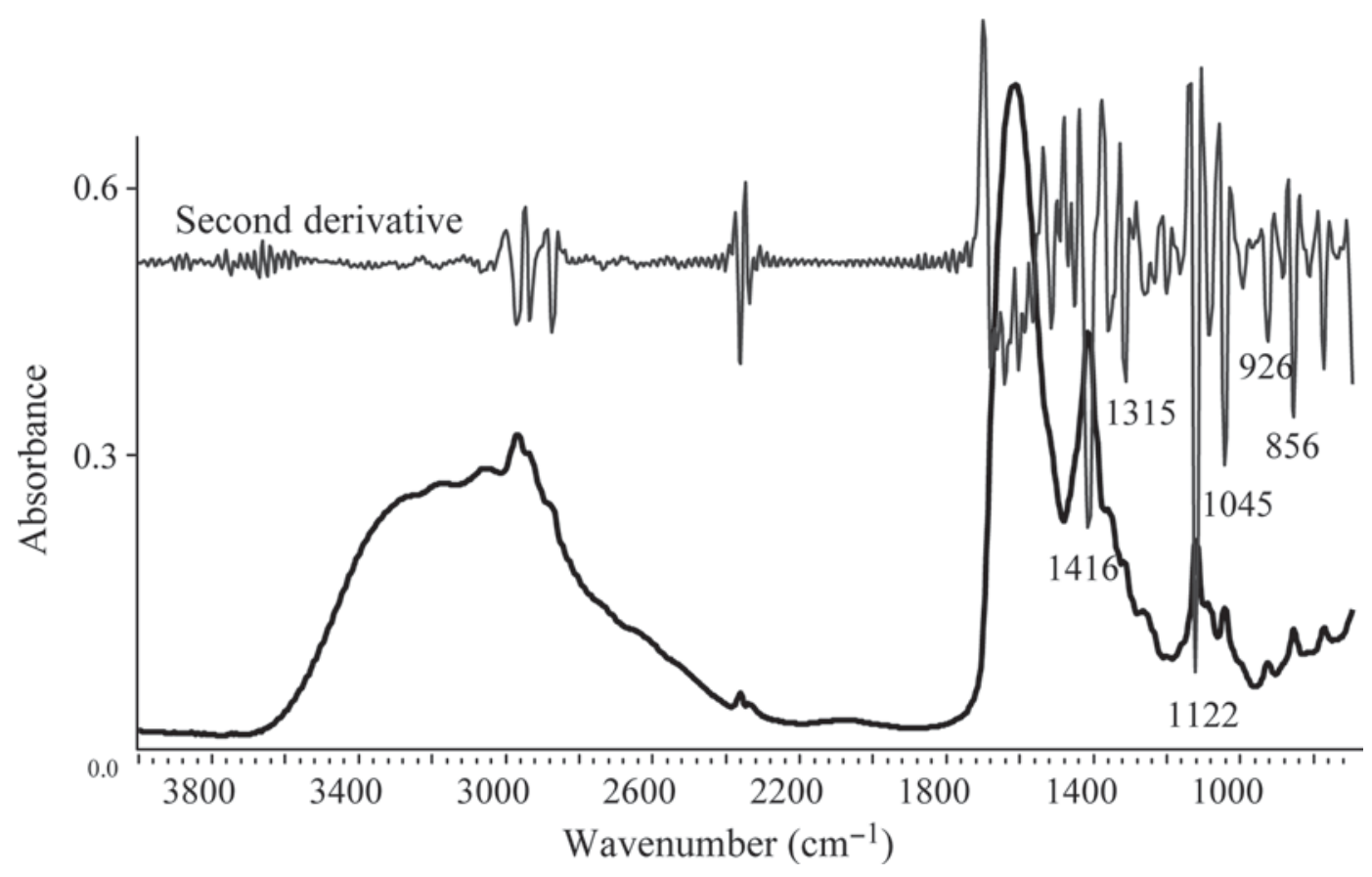

Figure 1. Representative spectra of a water-soluble cheese extract by reflectance infrared microspectroscopy in the range of 4,000 to 700 $\mathrm{cm}^{-1}$.

Interestingly, only the replicated samples for the cheeses manufactured without adjunct cultures formed 2 distinct clusters (ICD >30) after ripening for $90 \mathrm{~d}$, indicating that the 2 replicates developed very different chemical compositions during the ripening process (Table 1). Interclass distances are a measurement of the separation between classes (Vogt and Knutsen, 1985), and ICD values greater than 3.0 are considered to be sufficient for the 2 groups to be discriminated (Kvalheim and Karstang, 1992). These compositional differences could be attributed to the presence of adventitious NSLAB that may come from the raw milk

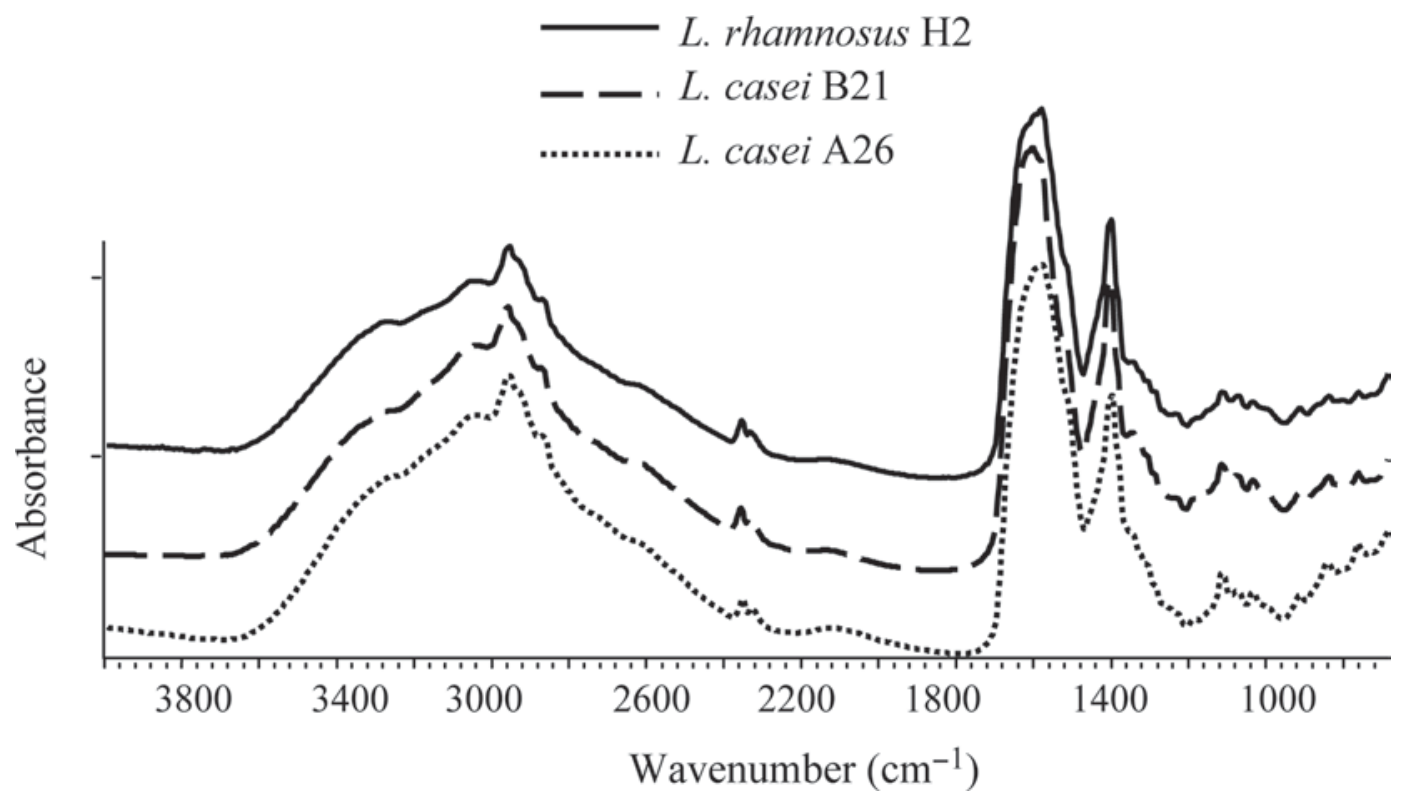

Figure 2. Representative spectra of water-soluble extracts of cheese samples manufactured with 3 Lactobacillus adjunct strains, Lactobacillus casei A26, L. casei B21, and Lactobacillus rhamnosus, after $90 \mathrm{~d}$ of ripening at the spectral region of 4,000 to $700 \mathrm{~cm}^{-1}$. 
A.

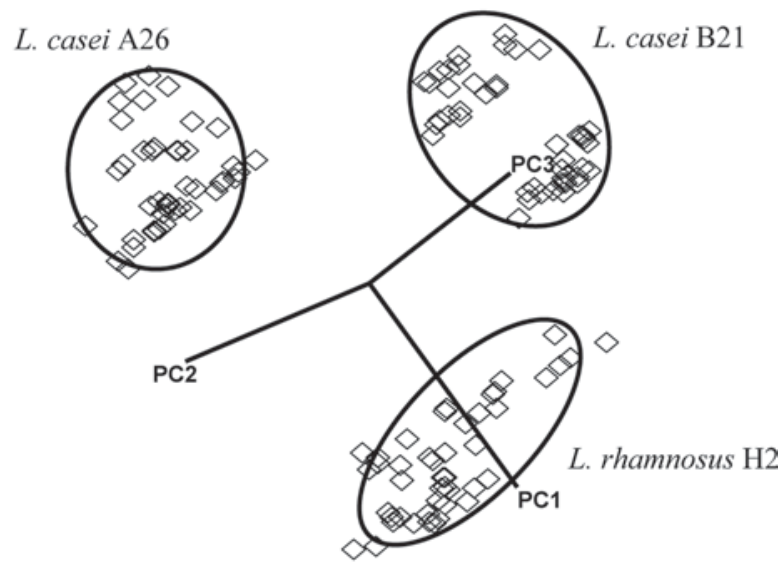

B.

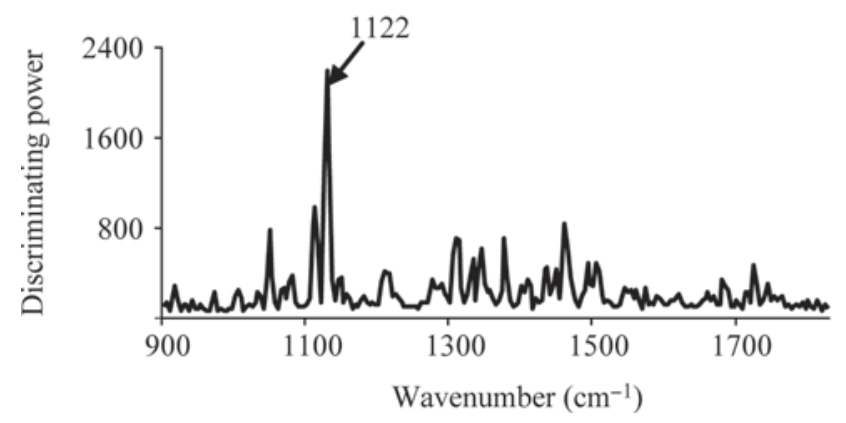

Figure 3. Soft independent modeling of class analogy (SIMCA) analysis (A) and a SIMCA discriminating power plot (B) of cheese samples with adjunct Lactobacillus casei A26, L. casei B21, and Lactobacillus rhamnosus after $90 \mathrm{~d}$ of ripening. $\mathrm{PC}=$ principal component.

and from postpasteurization contamination through the factory environment (Fox et al., 2000). A small number of NSLAB may survive pasteurization, grow during cheese ripening (Jordan and Cogan, 1999), and dominate the microbial population in cheese for much of the ripening period (Williams et al., 2000). Crow et al. (2001) suggested that using high levels of desirable NSLAB in cheese throughout ripening would provide balanced flavor reactions and minimize the undesirable effects of adventitious NSLAB by competition of the energy source.

The SIMCA discriminating power plots (Figure 3B) were used to determine the wavenumbers responsible for explaining most of the variances among classes that were having a predominant effect on the sample classification after $90 \mathrm{~d}$. The band at $1,122 \mathrm{~cm}^{-1}$, possibly associated with asymmetric-symmetric stretching vibrations of S-O from sulfur-oxy compounds (Coates, 2000; Sun and Wang, 2006), was largely responsible for discriminating cheeses manufactured with different adjunct cultures after $90 \mathrm{~d}$ of ripening (Figure 3B).
Eliardi (2008) also reported that this band is important in differentiating commercially manufactured Swiss cheeses.

At the end of $90 \mathrm{~d}$ of ripening, cheeses manufactured with or without adjunct cultures showed comparable discriminating profiles with similar band intensities (Figure 4). Major absorption bands were observed at $1,412,1,431,1,481$, and $1,705 \mathrm{~cm}^{-1}$, associated with asymmetric stretching of the carboxylic group (Agnihotri et al., 2006), $\mathrm{CH}_{2}$ symmetric bending (Ouajai and Shanks, 2005; Polovka et al., 2006), $\mathrm{C}=\mathrm{C}$ from the aromatic group (Loo et al., 2008), and the $\mathrm{C}=\mathrm{O}$ stretching vibration (Barth, 2000; Ravat et al., 2000), respectively.

Cheeses manufactured with L. casei A26, as well as the cheese manufactured without an adjunct, showed an absence of the band at $1,520 \mathrm{~cm}^{-1}$, which Coates (2000) associated with aromatic nitrogen-containing compounds. Kocaoglu-Vurma et al. (2008) reported that cheese samples manufactured with adjunct $L$. casei A26 received low sensory scores in cooked/milky flavor and in aromatics associated with cooked milk when compared with cheeses manufactured with adjunct $L$. casei B21 and L. rhamnosus H2. It is well established that strain dependence of adjunct cultures has an effect on cheese flavor and chemistry (Crow et al., 2001), yet the mechanism by which strains affect the flavor requires further exploration.

\section{Changes in Swiss Cheese During Ripening}

The SIMCA multivariate analysis was used to provide insight into the chemical changes occurring during the 90-d ripening process for cheeses manufactured with different adjunct Lactobacillus spp. The results suggest that Fourier-transform infrared microspectroscopy combined with multivariate analysis possessed the ability to classify cheese samples at different ripening periods (Figure 5, Table 1).

The SIMCA class projection showed similar clustering patterns for cheeses manufactured with adjunct cultures based on ripening time (Figure 5). The cheeses manufactured with no adjuncts exhibited more spectral variability and wider SIMCA clusters among replicated samples at later stages of ripening (d 30 to 90). For all cheeses manufactured with adjunct cultures, the largest ICD were observed between 1 and $90 \mathrm{~d}$ of ripening (Table 1). Interestingly, the SIMCA class projection showed that, for all treatments, marked changes in cheese chemistry occurred between 6 and $30 \mathrm{~d}$ of ripening (Figure 5). The d 6 to d 30 period represents the time the cheeses are in the warm-room ripening phase, when Propionibacterium freudenreichii converts lactate produced by lactic acid bacteria to propionic acid, ace- 


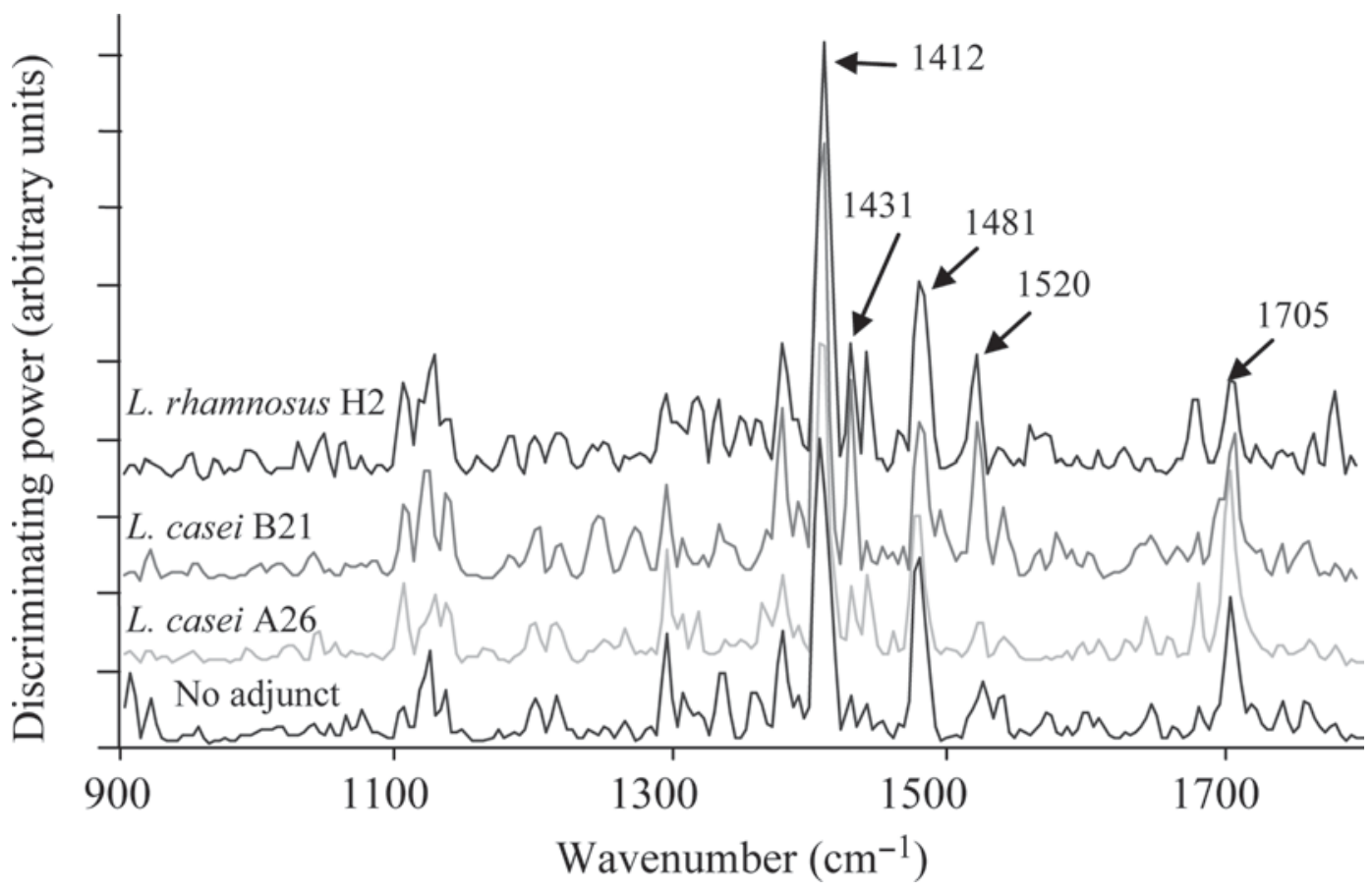

Figure 4. Soft independent modeling of class analogy (SIMCA) discriminating power plot of cheese samples with adjunct Lactobacillus casei A26, L. casei B21, or Lactobacillus rhamnosus, or without adjunct culture during ripening (1 to $90 \mathrm{~d}$ ).

Table 1. Interclass distances of cheeses with adjunct cultures Lactobacillus casei A26 (A), L. casei B21 (B), Lactobacillus rhamnosus $\mathrm{H} 2(\mathrm{C})$, and no adjunct (D) at different ripening days based on soft independent modeling of class analogy class projection of the Fourier-transform infrared microspectroscopy spectra collected in the 900 to $1,800 \mathrm{~cm}^{-1}$ region

\begin{tabular}{|c|c|c|c|c|c|}
\hline Item & Day 1 & Day 6 & Day 30 & Day 60 & Day 90 \\
\hline \multicolumn{6}{|l|}{ A } \\
\hline Day 1 & 0 & & & & \\
\hline Day 6 & $4.66^{1}$ & 0 & & & \\
\hline Day 30 & 39.83 & $36.7^{1}$ & 0 & & \\
\hline Day 60 & 37.83 & 34.33 & $6.47^{1}$ & 0 & \\
\hline Day 90 & 60.49 & 57.57 & 20.99 & $12.16^{1}$ & 0 \\
\hline \multicolumn{6}{|l|}{ B } \\
\hline Day 1 & 0 & & & & \\
\hline Day 6 & $5.59^{1}$ & 0 & & & \\
\hline Day 30 & 48.64 & $44.43^{1}$ & 0 & & \\
\hline Day 60 & 55.17 & 50.16 & $6.32^{1}$ & 0 & \\
\hline Day 90 & 71.58 & 65.17 & 13.32 & $11.36^{1}$ & 0 \\
\hline \multicolumn{6}{|l|}{$\mathrm{C}$} \\
\hline Day 1 & 0 & & & & \\
\hline Day 6 & $5.11^{1}$ & 0 & & & \\
\hline Day 30 & 50.39 & $48.48^{1}$ & 0 & & \\
\hline Day 60 & 50.74 & 48.48 & $12.09^{1}$ & 0 & \\
\hline Day 90 & 66.92 & 65.59 & 14.50 & $5.57^{1}$ & 0 \\
\hline \multicolumn{6}{|l|}{$\mathrm{D}$} \\
\hline Day 1 & 0 & & & & \\
\hline Day 6 & $4.57^{1}$ & 0 & & & \\
\hline Day 30 & 29.26 & $27.45^{1}$ & 0 & & \\
\hline Day 60 & 64.75 & 64.88 & $28.49^{1}$ & 0 & \\
\hline Day 90 & 35.21 & 33.41 & 15.86 & $18.67^{1}$ & 0 \\
\hline
\end{tabular}

${ }^{1}$ Indicates interclass distances between 2 conjunct ripening periods. 

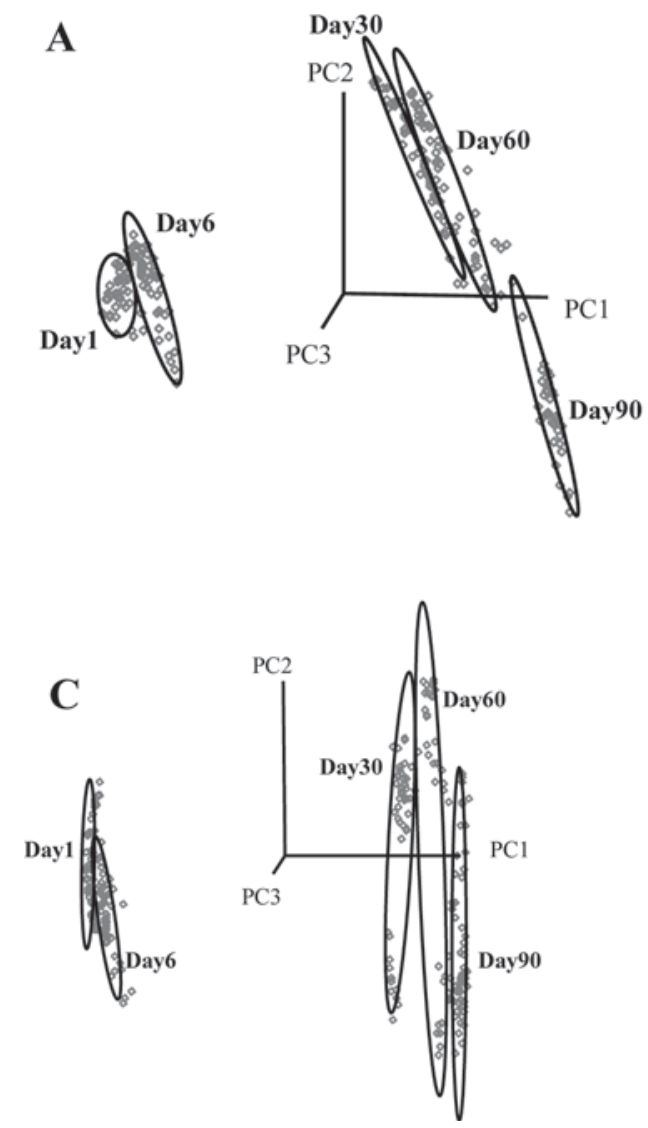
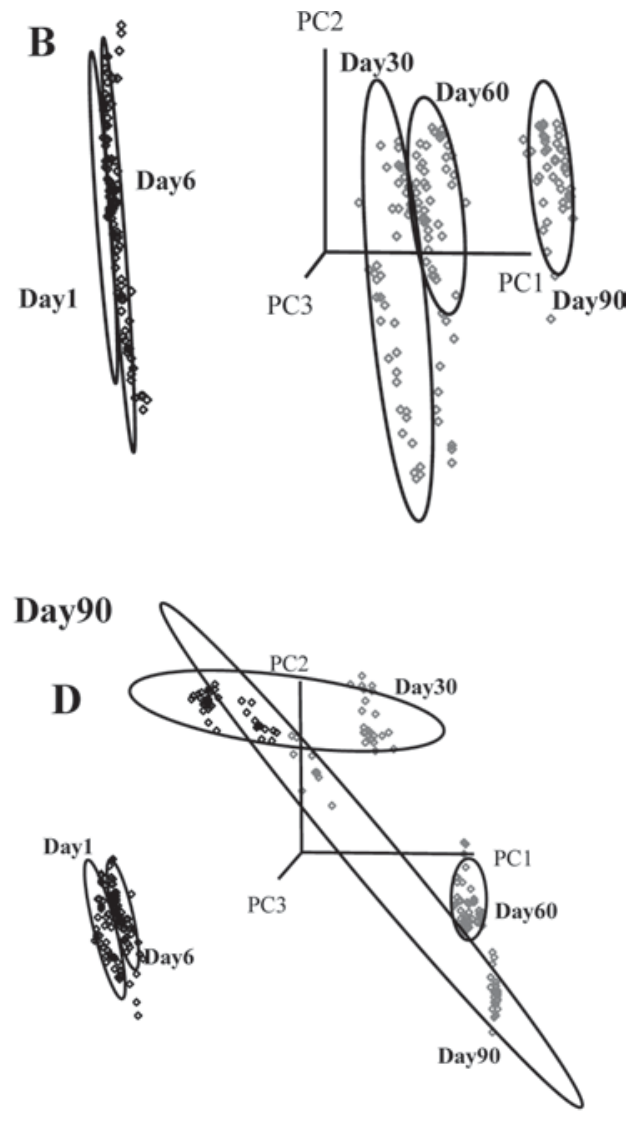

Figure 5. Soft independent modeling of class analogy (SIMCA) class projections of Swiss cheese samples manufactured with adjunct Lactobacillus casei A26 (A), L. casei B21 (B), or Lactobacillus rhamnosus H2 (C), or without adjunct culture (D) at different ripening days. PC $=$ principal component.

tic acid, and carbon dioxide. The differences in chemical composition of cheese samples in the warm-room period have also been associated with proteolysis (Valence et al., 2000), leading to the fastest rate of accumulation of total free AA (Kocaoglu-Vurma et al., 2008).

During the ripening time intervals, the largest ICD for the cheeses cultured with different adjunct cultures occurred between d 6 and 30 (ICD >36; Table 1), confirming our previous observation that most of the changes in chemical composition occurred in the warmroom ripening stage (Figure 5). In addition, cheeses manufactured with $L$. casei adjunct cultures showed more pronounced differences in composition between d 60 and 90 of ripening (ICD 12.16 and 11.36 for $L$. casei A26 and B21, respectively) as compared with $L$. rhamnosus (ICD 5.57). On the contrary, cheese manufactured with $L$. rhamnosus showed higher ICD between d 30 and 60 of ripening (ICD 12.09) as compared with L. casei (ICD 6.47 and 6.32 for L. casei A26 and B21, respectively; Table 1). Kocaoglu-Vurma et al. (2008) reported that L. rhamnosus has a slower rate of consumption of citric acid compared with L. casei A26 and
L. casei $\mathrm{B} 21$. By the end of d 30 of ripening, the citric acid was depleted for cheeses with $L$. casei A26 and $L$. casei B21 as adjunct cultures, whereas cheese with $L$. rhamnosus $\mathrm{H} 2$ still contained citric acid $>0.1 \mathrm{mg} / \mathrm{g}$ of cheese (Kocaoglu-Vurma et al., 2008).

The discriminating power of all 3 cheeses manufactured with adjunct cultures at different ripening periods was analyzed. A representative discriminating power for cheese manufactured with $L$. rhamnosus $\mathrm{H} 2$ is shown in Figure 6 . The highest intensity in the discriminating power plot was observed between d 6 and 30, indicating the extent of chemical changes that happened during this ripening period. The band at $1,412 \mathrm{~cm}^{-1}$ had the greatest contribution to the discrimination and has been associated with asymmetric and symmetric stretching vibrations of the carboxylate (COO-) group from acidic AA (glutamic or aspartic acids; Barth, 2000; Tayfur and Guner, 2005; Agnihotri et al., 2006).

The lowest discriminating power was found between d 1 and 6 of ripening, indicating that the changes in chemical composition were relatively slow during the initial cooling period compared with the latter ripening 


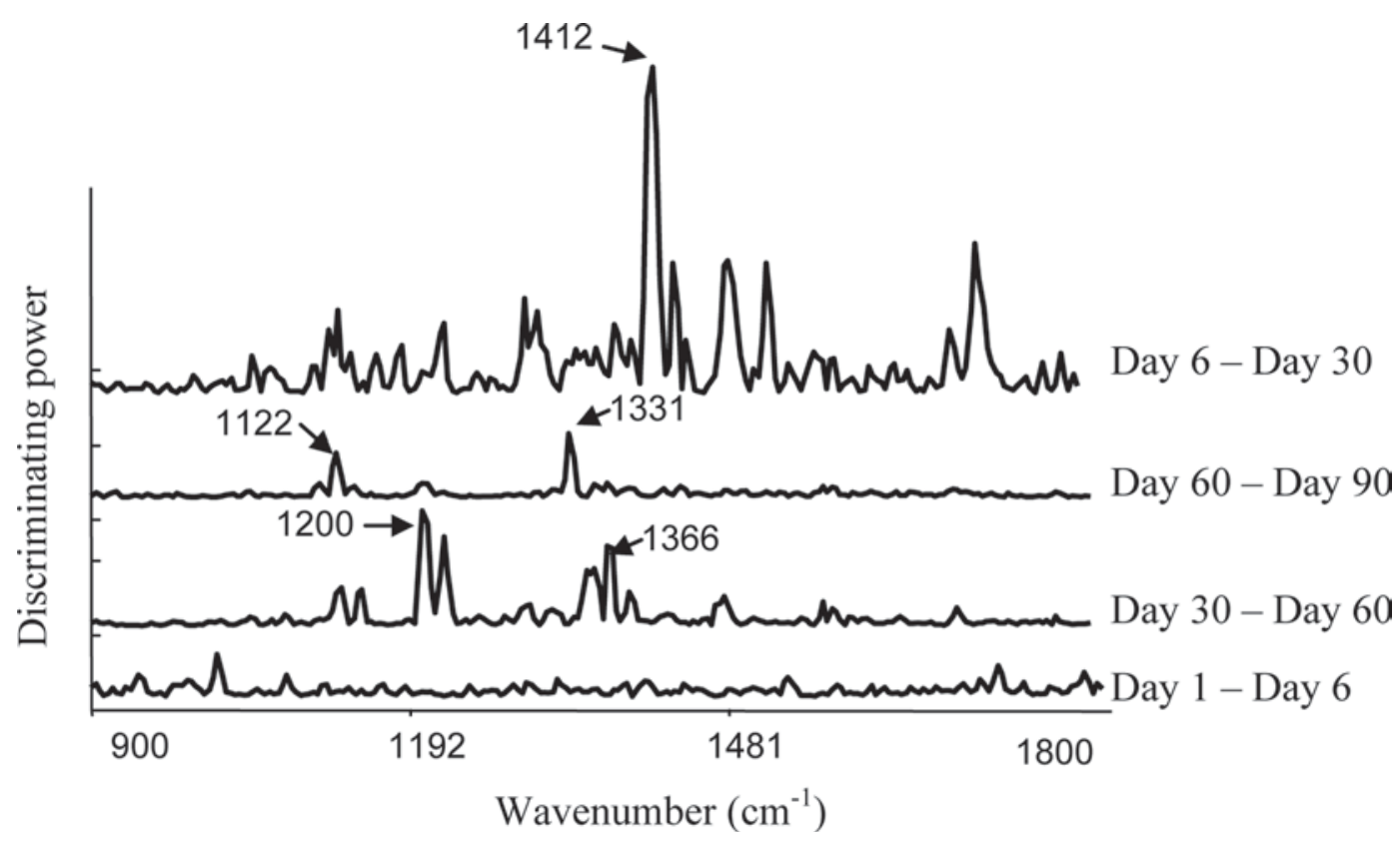

Figure 6. Soft independent modeling of class analogy (SIMCA) discriminating power plots depicting changes during ripening of Swiss cheeses manufactured with adjunct culture of Lactobacillus rhamnosus $\mathrm{H} 2$.

stages. Similar results were also documented by Valence et al. (2000), who reported that proteolysis remained at a low level during cold-room ripening and increased dramatically during warm-room ripening.

The predominant bands that were mainly responsible for the discrimination and assignment of the associated functional groups are compiled in Table 2. The symmetric stretching vibration of $\mathrm{COO}-$ at $1,412 \mathrm{~cm}^{-1}$ as the most predominant band over ripening was considered because of the considerable increase in proteolysis at the warm-room stage. During the ripening period between $\mathrm{d} 30$ and 60 , the increase in the band at 1,200 $\mathrm{cm}^{-1}$ caused by the stretching vibration of $-\mathrm{C}-\mathrm{O}$ from lipids (Guillen and Cabo, 1997) was associated with lipolysis. Proteolysis and lipolysis are the 2 major biochemical processes during ripening that contribute to the flavor of most cheese varieties by the production of fatty acids, AA and their derivatives, and other organic compounds (Park and Drake, 2005). During the ripening period between d 60 and 90, predominant bands were found at $1,122,1,331$, and $1,335 \mathrm{~cm}^{-1}$. These wavenumbers were associated with the asymmetric-symmetric stretching vibration from sulfur-containing compounds (Coates, 2000; Sun and Wang, 2006). The release of these sulfur-containing compounds at the later ripening stages has been associated with intensifying the flavor characteristics of cheese (Weimer et al., 1999).

\section{CONCLUSIONS}

The water-soluble fractions from Swiss cheese manufactured with different adjunct cultures were evaluated

Table 2. Assignments of major infrared bands during the ripening process of cheeses ${ }^{1}$

\begin{tabular}{llll}
\hline Ripening period & \multicolumn{1}{c}{ Band frequency, $\mathrm{cm}^{-1}$} & Possible functional group assignment & Reference \\
\hline Day 6 to 30 & $1,412^{2}$ & $\nu_{\mathrm{s}}(\mathrm{COO}-)$ & Agnihotri et al. (2006) \\
& 1,431 & $\delta(\mathrm{CH})$ & Ouajai and Shanks (2005); Polovka et al. (2006) \\
& 1,481 & $\nu(\mathrm{C}=\mathrm{C})$ from aromatic group & Loo et al. (2008) \\
& $1,697,1,705$ & $\nu(\mathrm{C}=\mathrm{O})$ & Barth (2000); Ravat et al. (2000) \\
Day 30 to 60 & $1,200,{ }^{2} 1,207^{2}$ & $\nu(-\mathrm{C}-\mathrm{O})$ & Guillen and Cabo (1997) \\
& $1,385,1,350,1,366$ & $\delta \mathrm{s}\left(\mathrm{CH}_{3}\right)$ & Barth $(2000)$ \\
Day 60 to 90 & $1,122^{2}$ & Asymmetric-symmetric $\nu(\mathrm{S}-\mathrm{O})$ & Coates $(2000) ;$ Sun and Wang (2006) \\
& $1,331,{ }^{2} 1,335^{2}$ & from sulfur-oxy compounds & Coates $(2000)$ \\
& Asymmetric-symmetric $\nu(\mathrm{S}-\mathrm{O})$ & \\
\hline
\end{tabular}

\footnotetext{
${ }^{1} \nu=$ stretching vibration; $\delta=$ in-plane bending vibration; $\delta \mathrm{s}=$ symmetric in-plane bending vibration.
}

${ }^{2}$ Indicates the highest discriminating power for the ripening period. 
at $\mathrm{d}$ 1, 6, 30, 60, and 90 of ripening by Fourier-transform infrared microspectroscopy and multivariate analysis. Cheeses manufactured with adjunct cultures showed more uniform and consistent spectral profiles, leading to the formation of tight clusters by pattern-recognition analysis (SIMCA) as compared with cheeses with no adjuncts, which exhibited more spectral variability among the replicated samples. The adjunct cheeses were differentiated from each other predominantly based on the effect of sulfur-containing compounds observed at $1,122 \mathrm{~cm}^{-1}$. The greatest changes in the chemical profile of each cheese occurred during warm-room ripening between $\mathrm{d} 6$ and 30 . The band at 1,412 $\mathrm{cm}^{-1}$, which was associated with asymmetric and symmetric stretching of the carboxylate group of acidic AA, had the greatest contribution to differentiation. Interestingly, cheeses manufactured with $L$. casei adjunct cultures showed more pronounced differences in composition between $\mathrm{d}$ 60 and 90 of ripening as compared with L. rhamnosus. A high-throughput infrared microspectroscopy technique was developed that can further the understanding of biochemical changes occurring during the ripening process and serve as a rapid tool for the identification of marker bands by which Swiss cheese can be classified.

\section{REFERENCES}

Agnihotri, S. A., S. S. Jawalkar, and T. M. Aminabhavi. 2006. Controlled release of cephalexin through gellan gum beads: Effect of formulation parameters on entrapment efficiency, size, and drug release. Eur. J. Pharm. Biopharm. 63:249-261.

Barth, A. 2000. The infrared absorption of amino acid side chains. Prog. Biophys. Mol. Biol. 74:141-173.

Byler, D. A., and H. M. Farrell Jr. 1989. Infrared spectroscopic evidence for calcium ion interaction with carboxylate groups of casein. J. Dairy Sci. 72:1719-1723.

Chen, M., and J. Irudayaraj. 1998. Sampling technique for cheese analysis by FT-IR spectroscopy. J. Food Sci. 63:96-99.

Coates, J. 2000. Interpretation of infrared spectra, a practical approach. Pages 10815-10837 in Encyclopedia of Analytical Chemistry. R. A. Meyers, ed. John Wiley and Sons Ltd., Chichester, UK.

Crow, V., B. Curry, and M. Hayes. 2001. The ecology of nonstarter lactic acid bacteria (NSLAB) and their use as adjuncts in New Zealand Cheddar. Int. Dairy J. 11:275-283.

De Maesschalck, R., A. Candolfi, D. L. Masart, and S. Heuerding. 1999. Decision criteria for soft independent modeling of class analogy applied to near infrared data . Chemom. Intell. Lab. Syst. 47:65-77.

De Maesschalck, R., D. Jouan-Rimbaud, and D. L. Massart. 2000. The Mahalanobis distance. Chemom. Intell. Lab. Syst. 50:1-18.

Diem, M., M. Romeo, C. Matthaus, M. Miljkovic, L. Miller, and P. Lasch. 2004. Comparison of Fourier transform infrared (FTIR) spectra of individual cells acquired using synchrotron and conventional sources. Infrared Phys. Technol. 45:331-338.

Dunn, W. J., III, and S. Wold. 1995. SIMCA pattern recognition and classification. Pages 179-193 in Chemometric Methods in Molecular Design. H. van de Waterbeemd, ed. VCH Publishers, New York, USA.

Eliardi, A. 2008. Rapid profiling of Swiss cheese by mid-infrared/ attenuated total reflectance spectroscopy. MS Thesis. The Ohio State Univ., Columbus.

Engels, W. J. M., R. Dekker, C. De Jong, R. Neeter, and S. Visser. 1997. A comparative study of volatile compounds in the water- soluble fraction of various types of ripened cheese. Int. Dairy J. $7: 255-263$.

Fox, P. F., T. P. Guinee, T. M. Cogan, and P. L. H. McSweeney. 2000. Biochemistry of Cheese Ripening. Pages 236-257 in Fundamentals of Cheese Science. Aspen Publishers Inc., Gaithersburg, MD.

Fox, P. F., T. K. Singh, and P. L. H. McSweeney. 1994. Proteolysis in cheese during ripening. Pages 1-13 in Biochemistry of Milk Products. A. T. Andrews and J. R. Varley, ed. Woodhead Publishing Ltd., Cambridge, UK.

Gorostiza, A., A. J. Cichoscki, A. T. Valduga, E. Valduga, A. Bernardo, and J. M. Fresno. 2004. Changes in soluble nitrogenous compounds, caseins and free amino acids during ripening of artisanal Prato cheese; a Brazilian semi-hard cow's variety. Food Chem. 85:407-414.

Guillen, M. D., and N. Cabo. 1997. Characterization of edible oils and lard by Fourier transform infrared spectroscopy. Relationships between composition and frequency of concrete bands in the fingerprint region. J. Am. Oil Chem. Soc. 74:1281-1286.

Jordan, K. N., and T. M. Cogan. 1999. Heat resistance of Lactobacillus spp. isolated from Cheddar cheese. Lett. Appl. Microbiol. 29:136140

Kansiz, M., J. R. Gapes, D. McNaughton, B. Lendl, and K. C. Schuster. 2001. Mid infrared spectroscopy coupled to sequential injection analysis for the online monitoring of the acetone-butanol fermentation process. Anal. Chim. Acta 438:175-186.

Kieronczyk, A., S. Skeie, T. Langsrud, and M. Yvon. 2003. Cooperation between Lactococcus lactis and nonstarter lactobacilli in the formation of cheese aroma from amino acids. Appl. Environ. Microbiol. 69:734-739.

Koca, N., L. E. Rodriguez-Saona, W. J. Harper, and V. B. Alvarez. 2007. Application of Fourier transform infrared spectroscopy for monitoring short-chain free fatty acids in Swiss cheese. J. Dairy Sci. 90:3596-3603.

Kocaoglu-Vurma, N. A., W. J. Harper, M. A. Drake, and P. D. Courtney. 2008. Microbiological, chemical, and sensory characteristics of Swiss cheese manufactured with adjunct Lactobacillus strains using a low cooking temperature. J. Dairy Sci. 91:2947-2959.

Kvalheim, O. M., and T. V. Karstang. 1992. SIMCA - Classification by means of disjoint cross-validated principal components models. Page 209-248 in Multivariate Pattern Recognition in Chemometrics: Illustrated by Case Studies. R. G. Breton, ed. Elsevier, Amsterdam, the Netherlands.

Langsrud, T., and G. M. Reinbold. 1973. Flavor development and microbiology of Swiss cheese-A review: III. Ripening and flavor production. J. Milk Food Technol. 36:593-609.

Lavine, B. K. 2000. Clustering and classification of analytical data. Pages 1-21 in Encyclopedia of Analytical Chemistry. R. A. Meyers, ed. John Wiley and Sons, New York, NY.

Law, B. A. 2001. Controlled and accelerated cheese ripening: The research base for new technologies. Int. Dairy J. 11:383-398.

Loo, A. Y., K. Jain, and I. Darah. 2008. Antioxidant activity of compounds isolated from the pyroligneous acid, Rhizophora apiculata. Food Chem. 107:1151-1160.

Lynch, C. M., P. L. H. McSweeney, P. F. Fox, T. M. Cogan, and F. D. Drinan. 1997. Contribution of starter lactococci and non-starter lactobacilli to proteolysis in Cheddar cheese with a controlled microflora. Lait 77:441-459.

Männig, A., N. A. Baldauf, L. A. Rodriguez-Romo, A. E. Yousef, and L. E. Rodriguez-Saona. 2008. Differentiation of Salmonella enterica serovars and strains in cultures and food using infrared spectroscopic and microspectroscopic techniques. J. Food Prot. $71: 2249-2256$.

Martin-del-Campo, S. T., D. Picque, R. Cosio-Ramirez, and G. Corrieu. 2007. Middle infrared spectroscopy characterization of ripening stages of Camembert-type cheeses. Int. Dairy J. 17:835-845.

Ngo-Thi, N. A., C. Kirschner, and D. Naumann. 2003. Characterization and identification of microorganisms by FT-IR microspectrometry. J. Mol. Struct. 661:371-380.

Noël, Y., P. Poyoval, A. Thierry, V. Gagnaire, and R. Grappin. 1999. Eye formation and Swiss-type cheeses. Pages 222-250 in Technology of Cheese Making. B. A. Law, ed. CRC Press, Boca Raton, FL. 
Ouajai, S., and R. A. Shanks. 2005. Composition, structure and thermal degradation of hemp cellulose after chemical treatments. Polym. Degrad. Stabil. 89:327-335.

Park, Y., and M. A. Drake. 2005. Effect of 3 months frozen-storage on organic acid contents and sensory properties, and their correlations in soft goat milk cheese. Small Rumin. Res. 58:291-298.

Polovka, M., J. Polovkova, K. Vizarova, S. Kirschnerova, L. Bielikova, and M. Vrska. 2006. The application of FTIR spectroscopy on characterization of paper samples, modified by Bookkeeper process. Vib. Spectrosc. 41:112-117.

Ravat, B., R. Gschwind, M. Grivet, E. Duverger, A. Chambaudet, and L. Makovicka. 2000. Electron irradiation of polyurethane: Some FTIR results and a comparison with an EGS4 simulation. Nucl. Instrum. Methods Phys. Res. B 160:499-504.

Rodriguez-Saona, L. E., N. Koca, W. J. Harper, and V. B. Alvarez 2006. Rapid determination of Swiss cheese composition by Fourier transform infrared/attenuated total reflectance spectroscopy. J. Dairy Sci. 89:1407-1412.

Safar, M., P. R. Bertrand, M. F. Devaux, and C. Genot. 1994. Characterization of edible oils, butters, and margarines by Fourier transform infrared spectroscopy with attenuated total reflectance. J. Am. Oil Chem. Soc. 71:371-377.

Schmitt, J., and H. Flemming. 1998. FTIR spectroscopy in microbial and material analysis. Int. Biodeterior. Biodegrad. 41:1-11.

Singh, T. K., M. A. Drake, and K. R. Cadwallader. 2003. Flavor of cheddar cheese: A chemical and sensory perspective. Compr. Rev. Food Sci. Food Saf. 2:166-189.

Smith, B. C. 1998. Infrared Spectral Interpretation: A Systematic Approach. CRC Press, Boca Raton, FL.
Subramanian, A., W. J. Harper, and L. E. Rodriguez-Saona. 2009. Cheddar cheese classification based on flavor quality using a novel extraction method and Fourier transform infrared spectroscopy . J. Dairy Sci. 92:87-94.

Sun, S., and A. Wang. 2006. Adsorption kinetics of $\mathrm{Cu}(\mathrm{II})$ ions using N,O-carboxymethyl-chitosan. J. Hazard. Mater. 131:103-111.

Tayfur, M., and A. Guner. 2005. Adsorption of certain amino acids onto crosslinked diethylaminoethyl dextran microbeads. J. Appl. Polym. Sci. 96:1985-1991.

Valence, F., S.-M. Deutsch, R. Richoux, V. Gagnaire, and S. Lortal. 2000. Autolysis and related proteolysis in Swiss cheese for two Lactobacillus helveticus strains. J. Dairy Res. 67:261-271.

Vogt, N. B., and H. Knutsen. 1985. SIMCA pattern recognition classification of five infauna taxonomic groups using non-polar compounds analyzed by high resolution gas chromatography. Mar. Ecol. Prog. Ser. 26:145-156.

Weimer, B., K. Seefeldt, and B. Dias. 1999. Sulfur metabolism in bacteria associated with cheese. Antonie Leeuwenhoek 76:247261.

Wenning, M., H. Seiler, and S. Scherer. 2002. Fourier-transform infrared microspectroscopy, a novel and rapid tool for identification of yeasts. Am. Soc. Microbiol. 68:4717-4721.

Williams, A. G., S. E. Withers, and J. M. Banks. 2000. Energy sources of non-starter lactic acid bacteria isolated from Cheddar cheese. Int. Dairy J. 10:17-23.

Woodcock, T., C. C. Fagan, C. P. O'Donnell, and G. Downey. 2008. Application of near and mid-infrared spectroscopy to determine cheese quality and authenticity. Food Bioprocess. Technol. 1:117129 . 